\title{
CASO CLÍNICO.
}

\section{MANEJO DE DERMATITIS ATÓPICA CRÓNICA CON MEDICINA BIORREGULADORA}

\section{Médico tratante}

Díaz Forero Daniela Andrea

\section{Descripción del problema.}

\section{Dermatitis atópica}

La dermatitis atópica se reconoce cada vez más como una enfermedad multifactorial y heterogénea con diferentes fenotipos moleculares o celulares que caracterizan a diferentes poblaciones.

Los estudios continúan aclarando las interacciones clave entre los genes de susceptibilidad, los factores ambientales, el microbioma, la integridad de la barrera deteriorada y la desregulación inmunológica.

La identificación de subconjuntos inmunes, incluidos Th17, Th22 y Th9, ha cambiado los paradigmas de la enfermedad. de Th1 / Th2 bifásico conducido a una enfermedad compleja de múltiples ejes.

En la clínica se puede evaluar con escalas que miden las áreas corporales comprometidas en gravedad y síntomas del paciente, dos de ellas son:

- $\quad$ SCORAD (Severity scoring atopic dermatitis)

- $\quad$ EASI (Eczema Area and Severitylndex).

Los protocolos de manejo incluyen intervención farmacológica y no farmacológica en los que contiene los emolientes con urea y la fototerapia; dentro de los manejos farmacológicos encontramos los corticoides tópicos como pilar fundamental, seguido por inhibidores tópicos de la calcineurina. Se pueden encontrar intervenciones sistémicas con corticoides sistémicos, metrotexato, micofelonato, aziatropina, inhibidores selectivos de la interleucina 4 y 13 . Y en casos agudos el uso de antihistamínicos y si se documenta sobreinfección manejo antibiótico con cefalosporina de 1ra línea.

Fecha inicio: 01 marzo 2021

Información del paciente:

Género: femenina

Edad: 26 años

Fecha de nacimiento: 02/ mar/1995

Natural: Bogotá

Procedente: Bogotá

Ocupación: publicista

Motivo de Consulta: "no puedo más con la piel"

Paciente femenina de 28 años quien consulta por cuadro de larga data de lesiones cutáneas descamativas, pruriginosas, sensación urente al contacto con el agua y productos de aseo personal, predomina en región del cuello, tórax y abdomen, algunas lesiones se presentan en áreas de pliegues cutáneos. Importante alteración de la calidad de vida y autoestima. Factores exacerbantes días previos a sangrado menstrual, productos cosméticos, en una ocasión presentó exacerbación inmediata posterior a sesión en spa.

Factores atenuantes al finalizar el periodo menstrual.

Ha recibido manejo con corticoides sistémicos y tópicos con resolución momentánea de las lesiones, pero reincidencia de estas, cerca de 50 sesiones de fototerapia sin cambios importantes en las lesiones.

\section{Revisión por sistemas:}

Ojos: episodios de blefaritis

Orl: rinitis en la mañana con el frio.

Gastrointestinal: episodios de estreñimiento e intolerancia algunos alimentos y lácteos, deposiciones diarias no fétidas. Ginecológicos: cambio de leucorrea con la T, múltiples manejos como vaginosis sin mejoría; dismenorrea desde uso del DIU con predominio de mastalgia. 


\section{Antecedentes:}

Primogénita, padres casados, gestación deseada, nacimiento por parto natural.

Patológicos: dermatitis, rinitis, amigdalitis con frecuencia en la infancia.

Quirúrgicos: embarazo ectópico, resección de masas quísticas mamarias.

Farmacológicos: niega

Alérgicos: niega conocidos.

Tóxicos: niega

Traumas: niega

Ginecobstétricos: G2P1E1V1 planificación actual con DIU, previamente uso de implante subdérmico por 8 años suspendió por aparición de masas. Dismenorrea desde uso del DIU con predominio de mastalgia.

Emocionales: Embarazo a los 15 años no planeado, con llanto importante hasta los 6 meses, no emesis, ocasionalmente antojos de camarones.

\section{Factores del estilo de vida}

Sueño y relajación: 6 horas, sueño no reparador.

Movimiento y ejercicio: en el momento sedentaria, previo al inicio de la pandemia se movilizaba en bicicleta.

Nutrición e hidratación: no tolerancia a los lácteos, ingesta promedio de 1 lt de agua, consumo de carne de res y cerdo poco consumo de pollo y pescados, consumo promedio de frutas y verduras sin variación, consumo de café cada 4 días, consumo irregular de postres y dulces, consumo frecuente de pan, consumo de comida rápida cada 15-20 días.

Estrés: laboral, emocional, baja autoestima

Relaciones y red social: madre soltera, conflictos familiares, red de apoyo aceptable, sin pareja en el momento, frecuenta con amistades, buena relación con la jefe.

\section{Laboratorios}

17-mar-2021:

- Hemograma leucos 7.990 neutros 5.480 linfos 1.580 rojos 5.080 hb $15 \mathrm{~g}$ hto $45 \%$ plqts 278.000

- Tsh: $1.76 \mathrm{uUl} / \mathrm{ml}$

- IgE: $611 \mathrm{UI} / \mathrm{ml}$

Rast acaros $>100 \mathrm{UI} / \mathrm{ml}$ Positivo muy alto para alergeno clase IV: mayor o igual a $100 \mathrm{UI} / \mathrm{ml}$.

IgE específica pastos (ORCHARD GRASS) $3.95 \mathrm{UI} / \mathrm{m}$ Positivo alto para alergeno clase III: $3.50-17.49 \mathrm{UI} / \mathrm{ml}$.

IgE específica pastos (TIMOTY GRASS) $0.59 \mathrm{UI} / \mathrm{m}$ Positivo bajo para alergeno clase i: $0.35-3.49 \mathrm{UI} / \mathrm{ml}$.

IgE específica epitelio perro $1.56 \mathrm{UI} / \mathrm{ml}$ Positivo moderado para alergeno clase II: $0.70-3.49 \mathrm{UI} / \mathrm{ml}$.

IgE específica epitelio gato $1.15 \mathrm{UI} / \mathrm{ml}$ Positivo moderado para alergeno clase II: $0.70-3.49 \mathrm{UI} / \mathrm{ml}$.

IgE específica Blomia Tropicalis $14.20 \mathrm{UI} / \mathrm{ml}$ Positivo alto para alergeno clase II: $3.50-17.49 \mathrm{UI} / \mathrm{ml}$.

IgE específica Dermatophagoides farinae $92.30 \mathrm{UI} / \mathrm{ml}$ Positivo muy alto para alergeno clase III: 52.5 - $99.9 \mathrm{UI} / \mathrm{ml}$.

\section{$17-\operatorname{mar}-2021$}

Ecografía transvaginal: útero piriforme en anteversoflexión volumen $65.7 \mathrm{cc}$. Endometrio trilaminar de 5,5mm, en fase proliferativa del ciclo menstrual. Cavidad endometrial contiene DIU tipo T de cobre, cuyo extremo superior se ubica a $13.0 \mathrm{~mm}$ del fondo uterino. Ovario derecho de $8.2 \mathrm{cc}$ e izquierdo $5.8 \mathrm{cc}$ sin masas anexiales.

\section{3 - sept -2020}

Ecografía mamaria: Derecha quiste simple en coordenada 9.5 de $5 \mathrm{~mm}$. Izquierda nódulo sólido, ovalado, homogéneo y circunscrito, en coordenadas 3.2 de $13 \times 6 \times 13$ mm, volumen de 0.46 cc, de aspecto y comportamiento benigno.

Escalas de puntuación de la enfermedad. SCORAD 65.8 puntos (>50 puntos enfermedad grave)

EASI 20 puntos (12-20 puntos enfermedad moderada)

Examen físico

Signos vitales: TA 120/70 FC 80 lpm FR $16 \mathrm{rpm}$ T36 ${ }^{\circ} \mathrm{C}$ Peso 5 kg Talla: 168

Buen estado general, consciente, orientada, mucosas normocrómicas. Ojos con escleras claras. Bordes palpebrales con signos de blefaritis. Mucosa oral semihúmeda, sin lesiones, dientes con aparatos de ortodoncia. Cuello móvil sin masas palpables, contracturas musculares en región cervical. 
Tórax simétrico normo expansible, ruidos cardiacos rítmicos sin soplos, murmullo vesicular sin agregados. Abdomen con moderado panículo, no masas palpables, no irritación peritoneal.

Miembros superiores e inferiores con arcos de movilidad conservados, sin deformidades.

Piel se observa con signos de deshidratación crónica, aspera, rugosa, con múltiples áreas de eccema en parches con descamación, edema, eritema, excoriación, exudación, lesiones costrosas y estigmas de rascado distribuidas sobre un $30 \%$ de la circunferencia del cuello, $70 \%$ de la circunferencia toracoabdominal, en pliegues cutáneos de axilas, codos y región poplítea.
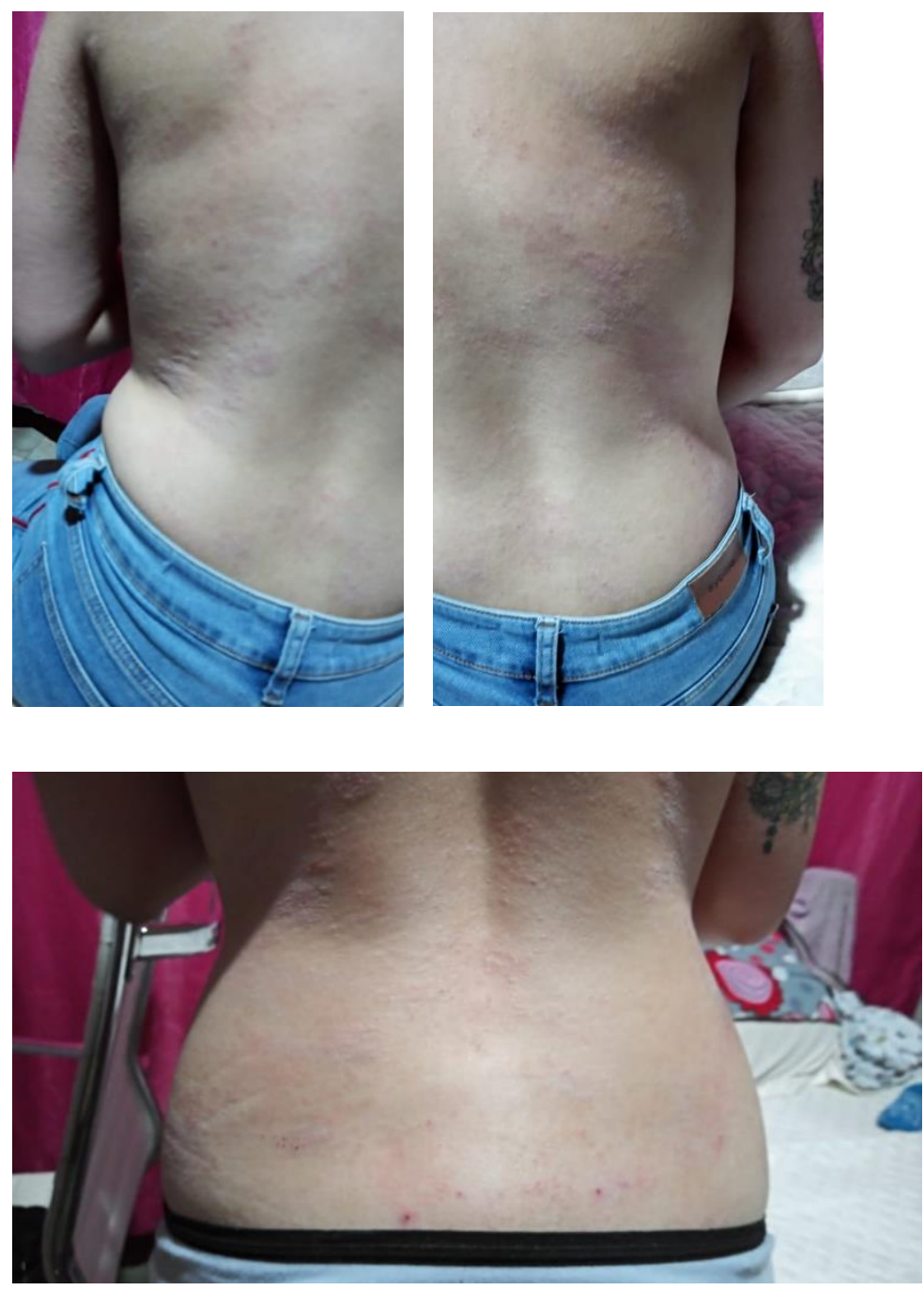

Tabla de progresión de la enfermedad desde la homotoxicología de la paciente de manejo.

\begin{tabular}{|c|c|c|c|}
\hline ECTODERMO & $\begin{array}{c}\text { FASE HUMORAL } \\
\text { Piel con primeras lesiones de } \\
\text { de neurotitis, aumento de la secreción } \\
\text { de nemsmisores y vagotonía }\end{array}$ & FASE MATRICIAL & FASE CELULAR \\
\hline
\end{tabular}




\begin{tabular}{|l|c|c|c|}
\hline ENDODERMO & $\begin{array}{c}\text { Alteración hormonas intestinales y } \\
\text { del eje hipotálamo hipófisis adrenal. }\end{array}$ & $\begin{array}{c}\text { Quistes mamarios con } \\
\text { calcificaciones mamarias } \\
\text { Mastopatía fibroquística }\end{array}$ & Embarazo ectópico \\
\hline MESODERMO & Hipermenorrea & Quistes ováricos & Ember \\
\hline
\end{tabular}

\section{Manejo biorregulador}

1. Dieta de eliminación durante 21 días. *

2. Suplementación con vitamina D oral en cápsulas de 7000 ui en 2 ocasiones y continuo de forma diaria con 2000 ui diarias

3. Suplementación con kyo-Dophilus

4. Dieta empieza a introducir alimentos al día 23 cada 3er día

5. Inicio de detoxificación y drenaje oral (10-mar-21)

- Nuxeel-Homaccord

- $\quad$ Berberis-Homaccord

- Lymphomyosot

6. Inicio de terapia dirigida y soporte orgánico

\begin{tabular}{|c|c|c|c|}
\hline FECHA & INMUNOMODULADOR & SOPORTE ORGÁNICO & VÍA \\
\hline \multirow[t]{3}{*}{24 marzo } & Traumeel s & Ubichinon compositum & sueroterapia \\
\hline & & Flamosin compositum & \\
\hline & & Hepar compositum & \\
\hline \multirow[t]{2}{*}{27 marzo** } & Traumeel s & Ubichinon compositum & Subcutáneo \\
\hline & & Coenzyme compositum & \\
\hline \multirow[t]{3}{*}{31 marzo } & Traumeel s & Hepar compositum & Sueroterapia \\
\hline & & Flamosin compositum & \\
\hline & & Cutis Compositum & \\
\hline \multirow[t]{3}{*}{10 abril } & Traumeel s & Flamosin compositum & sueroterapia \\
\hline & & Cutis Compositum & \\
\hline & & Ovarium compositum & \\
\hline \multirow[t]{7}{*}{17 abril } & Traumeel s & Hepar compositum & Puntos Ren \\
\hline & & Flamosin compositum & \\
\hline & & Cutis Compositum & \\
\hline & & Ovarium compositum & \\
\hline & & Ubichinon compositum & \\
\hline & & Coenzyme compositum & \\
\hline & & Hormeel snt gotas & oral \\
\hline \multirow[t]{6}{*}{25 abril } & Traumeel s & Hepar compositum & Puntos Ren \\
\hline & & Flamosin compositum & \\
\hline & & Cutis Compositum & \\
\hline & & Ovarium compositum & \\
\hline & & Ubichinon compositum & \\
\hline & & Coenzyme compositum & \\
\hline \multirow[t]{4}{*}{8 mayo } & - & Hepar compositum & Puntos Ren \\
\hline & & Flamosin compositum & \\
\hline & & Cutis Compositum & \\
\hline & & Ovarium compositum & \\
\hline \multirow[t]{4}{*}{16 mayo } & Traumeel s & Thyreoidea compositum & Puntos Ren \\
\hline & & Glándula Suprarrenalis suis injeel & \\
\hline & & Cutis Compositum & \\
\hline & & Ovarium compositum & \\
\hline 29 mayo & - & Thyreoidea compositum & Puntos Ren \\
\hline
\end{tabular}




\begin{tabular}{|c|c|c|c|}
\hline & & Cutis Compositum & \\
\hline & & Ovarium compositum & Puntos Ren \\
\hline 18 junio & - & Thyreoidea compositum & \\
\hline & & Cutis Compositum & Puntos Ren \\
\hline & - & Ovarium compositum & \\
\hline & & Thyreoidea compositum & \\
\hline & & Glándula Suprarrenalis suis injeel & \\
\hline & & Cutis Compositum & \\
\hline
\end{tabular}

* La dieta de eliminación se interrumpió al día 8 por que ingirió alimentos no permitidos (huevo) y se reinició hasta completar los 21 días.

** agudización sintomática.

7. Al control del 23 de marzo manifiesta mejoría sintomática importante con sensación de disminución de algunas de las lesiones.

8. El 27 marzo se presentó agudización de los síntomas y eccemas. Ver foto.

9. Al control del 10 de abril se ha iniciado la introducción de algunos alimentos con adecuada tolerancia.

10. Intervención emocional: el 25 de abril asistimos al parque Simón Bolívar en bicicleta y realizamos una sesión evacuatoria emocional, contacto directo con la naturaleza.

11. Control el 8 de mayo con mejoría importante de la calidad de vida, ganancia de peso, mejoría de la autoestima, se ajusta dieta.

12. Control 2 de julio la paciente está muy satisfecha con terapia instaurada.

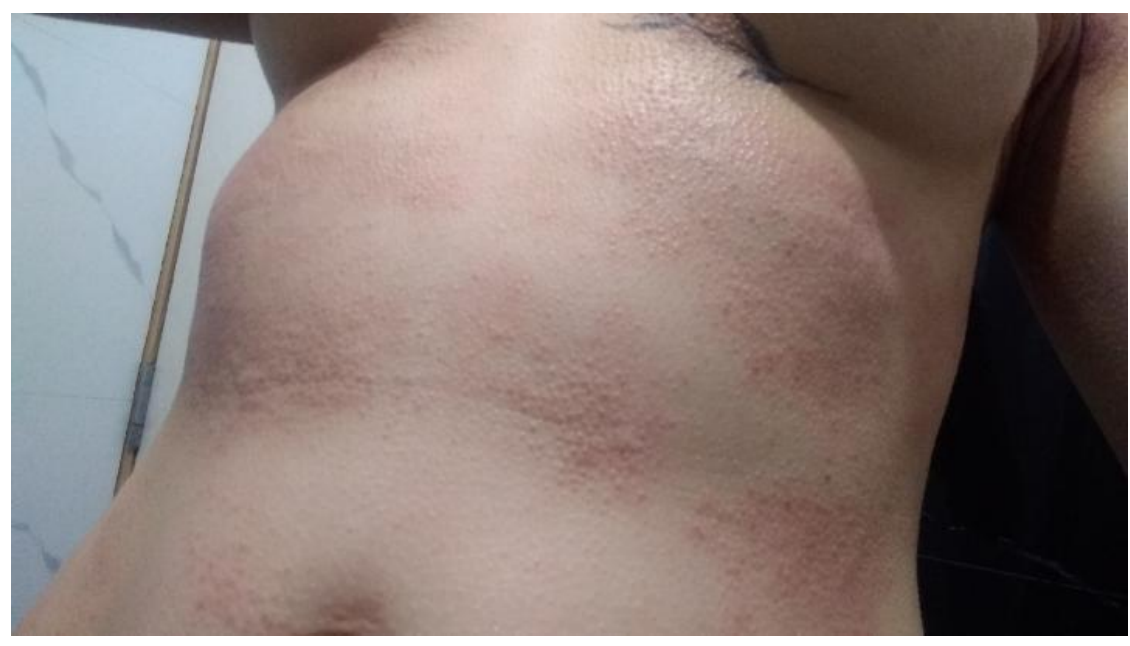



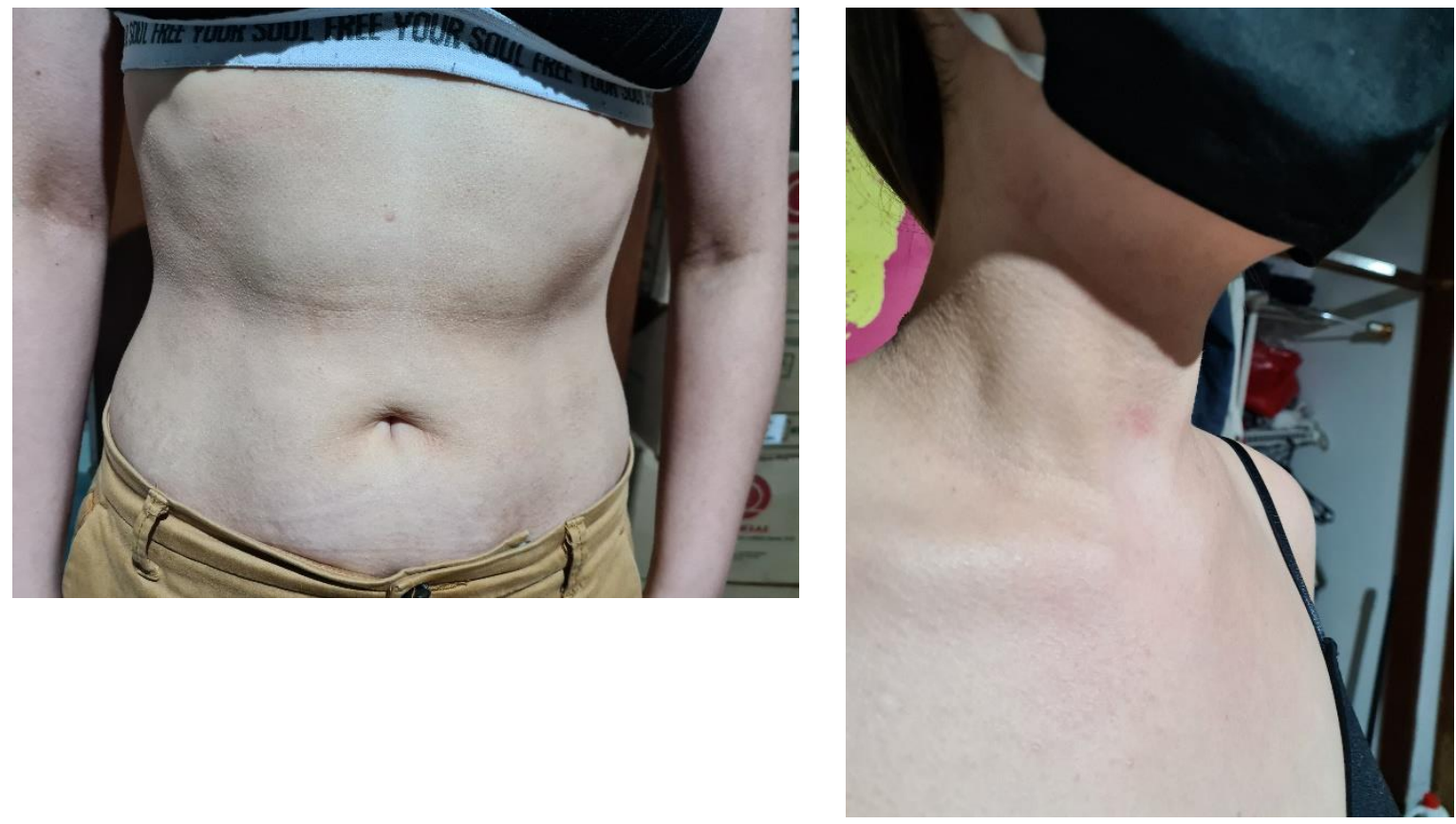

\section{Discusión y resultados}

La dermatitis atópica afecta la calidad de vida de la paciente y su autoestima limitando actividades que requieran exposición de la piel por vergüenza.

Hasta la fecha a pesar de los muchos avances no existe una terapia única que tenga un resultado ideal, satisfactorio y que se pueda sostener el tiempo en la medicina convencional.

El abordaje biorregulador tiene bases teóricas loables que pueden mostrar una luz en la intervención de este tipo de patologías y en la multicausalidad de esta, replicables y sostenibles en el tiempo para diferentes grupos etarios.

Se presenta un caso clínico en una fase cronificada, previamente intervenido con pocos resultados satisfactorios para la paciente; se encuentra en curso clínico con buena respuesta y evolución en el tiempo.

Se puede observar cómo los cambios en la alimentación especialmente la suplementación con diferentes micronutrientes y probióticos empieza a tener impacto temprano y cambios en la condición de la piel con disminución de prurito y eritema. Se presentó una reacción de agudización sintomática (bicameral) posterior a la primera sesión de sueroterapia Se documentó un cambio significativo por parte de la paciente cuando se inició la aplicación de medicamentos en puntos Ren y con la sesión evacuatoria en la naturaleza.

A la fecha los resultados son óptimos y prometedores para la paciente.

\section{Bibliografía}

1. Malik K, Heitmiller KD, Czarnowicki T. An update on the pathophysiology of Atopic Dermatitis. Dermatol Clin. 2017;35(3):317-326

2. Roessler A, Friedrich U, Vogelsang H, Bauer A, Kaatz M, Hipler UC, Schmidt I and Jahreis G: The immune system in healthy adults and patients with atopic dermatitis seems to be affected differently by a probiotic intervention. Clin Exp Allergy. 38:93-102. 2008. PubMed/NCBI

3. AsoColDerma- AC de D y CD, Centro dermatológico Federico Lleras Acosta, - IETS I de ET en S. Guía de práctica clínica (GPC) para el diagnóstico y tratamiento de la dermatitis atópica en Colombia. rev. asoc. colomb. dermatol. cir. dematol. [Internet]. 6 de octubre de 2020. Disponible en: https://revista.asocolderma.org.co/index.php/asocolderma/article/view/1508 
